\title{
Ubertiefte Talabschnitte im Berner Mittelland zwischen Alpen und Jura (Schweiz)
}

\author{
Christian SChlüChter *) \\ Valley, glacial erosion, neotectonics, basin of subsidence, channel, lacustrine sedimentation, \\ c-14 dating, continental quaternary Molasse Basin Switzerland.
}

K u rzf assung: Die übertieften Bereiche im Berner Querschnitt des Mittellandes zwischen Alpen und Jura werden, neben der Zungenbeckentheorie, vor dem Hintergrund neuer geophysikalischer Untersuchungsresultate aus den Schweizer Seen und den Resultaten des Landesnivellements beschrieben. Bedeutende Übertiefungen sind im Becken von Belp südlich von Bern mit ca. $270 \mathrm{~m}$, im seeländischen Trog bei Worben/Busswil mit ca. $285 \mathrm{~m}$ und im Unteren Emmental bei Gerlafingen mit mindestens $88 \mathrm{~m}$ durch Bohrungen belegt. Die Existenz einer basalen Erosionsrinne in der Molasse als Verbindung zwischen dem Becken von Belp mit dem seeländischen Trog oder dem Unteren Emmental ist fraglich. Zeitlich kann die maximale Tiefenerosion als sicher prae- oder frühriß-, stellenweise als mindelzeitlich (?) nach der klassischen Eiszeitchronologie angegeben werden. Zur genetischen Erklärung der übertieften Becken werden sowohl Gletschererosion (Zungenbeckenbildung/Cirque-Bildung im Alpenvorland) als auch krustendynamische Faktoren angeführt (Landesnivellement, Nachweis von Gasaustritten in einer Bohrung auf der Linie Gurten-nördliches Becken von Belp-Grauholz).

\section{["Overdeepened Basins" in the Molasse Foreland between the Alps and the Jura Mountains, Canton of Berne, Switzerland.]}

A bstract: The occurrence of so-called "overdeepened basins" in the Swiss Plain is discussed and their sedimentary Upper Pleistocene filling described. The old theory explaining the formation of the lakes along the border of the Alps by tectonic subsidence of the Alpine Front is compared with more recent results of geophysical investigations in Swiss lakes, with the results of precise levelling by the Swiss Topographic Survey and with glacial erosion in the Alpine Foreland. Considerable "overdeepening" is proved in the Belp Basin south of Berne (appr. 270 meters), in the Swiss Plain around Lyss-Worben/Busswil (appr. 285 meters) and in the Lower Emme Valley (minimum of 88 meters). A basal connection eroded in the Molasse sediments from the Belp Basin to the basin in the Swiss Plain ("seeländischer Trog") or to the Lower Emmental is not proven to exist. The base of the deepest eroded basins has been reached during pre- or early Riss, or during Mindel (?) at some places following the classical chronostratigraphic setting.

\section{In ha $1 \mathrm{ts}$ verzeich $\mathrm{n}$ is}

1. Forschungsgeschichtlicher Hintergrund

1.1. Historisches

1.2. Neue Forschungsergebnisse

2. Untersuchungsgebiete

2.1. Das Bernische Seeland

2.2. Das Untere Emmental

2.3. Das Aaretal Bern-Thunersee, insbesondere das Becken von Belp

3. Zusammenfassende Bemerkungen und Ausblick

4. Verdankung

5. Schriftenverzeichnis

*) Anschrift des Verfassers: Dr. Ch. S chlü chter, Institut für Grundbau und Bodenmechanik der ETH, Hönggerberg, CH-8093 Zürich, Schweiz. 


\section{Forschungsgeschichtlicher Hintergrund}

Eine Beschreibung der übertieften Talabschnitte im Berner Mittelland erfolgt mit Vorteil vor einem doppelschichtigen Hintergrund: einerseits vor einer Darstellung der historischen, wissenschaftsgeschichtlichen Entwicklung der Vorstellungen über Wesen und Genese der übertieften Talabschnitte im Gebiet der Schweiz, andererseits vor den Resultaten zeitgemäßer Forschungsprojekte zur Erforschung der Schweizer Seen und vor den Ergebnissen des Landesnivellements.

\subsection{Historisches}

Ausgehend von der Frage nach dem Ursprung der alpinen Randseen hat sich um die Jahrhundertwende eine zum Teil recht lebhaft geführte Kontroverse um die Wirksamkeit der Glazialerosion im allgemeinen, während der eiszeitlichen Gletschervorstöße ins Alpenvorland im speziellen, entwickelt. Vertreter der Theorie einer „eher schwach wirksamen Glazialerosion" (wie Heim 1885 und 1919, Gogarten 1910, mit einer vortrefflichen Zusammenstellung älterer Literatur) haben die Existenz der Alpenrandseen als Produkt einer jungen randalpinen Rücksenkung der tektonischen Alpenrandelemente erklärt. Die Alpenrandseen wären demnach Talabschnitte, die im eigenen Wasser ertrunken sind. Demgegenüber steht die Vorstellung von einer bedeutenden (Tiefen-)Erosion durch Gletscher, deren Vertreter, wie HeIm (1919: 13) schreibt, „die ungeheure Gletscherwirkung mit Schlagwörtern wie ,Übertiefung', ,Zungenbecken', ,Trogform', ,Stufenmündung' formuliert" haben (in der Schweiz u. a. FrueH 1906). Die Diskrepanz auf der Suche nach einer Erklärung, einerseits der Existenz der heutigen Alpenrandseen, andererseits der Ursache der primären Úbertiefung, also der Auskolkung des Felsuntergrundes auf die heute feststellbare Tiefe, ist bis heute nicht restlos überwunden. Allerdings hat die moderne Frage „wie sind die Seebecken strukturiert und wie sind die Sedimente aufgebaut?“, die alte Frage nach dem „warum sind die Seen gerade dort wo sie sind?“ etwas verdrängt. Ein Meilenstein in der historischen Entwicklung dieser Fragestellungen ist sicher die Toteistheorie von STAuB (1938). - Übertiefte Talabschnitte orographisch oberhalb und unterhalb der Alpenrandseen, die mit eiszeitlichen Lockergesteinen aufgefüllt sind, waren um die Jahrhundertwende weder genau lokalisiert, geschweige denn in ihrer Ausdehnung bekannt. Zudem macht ja die Theorie der tektonischen Alpenrandrücksenkung das Vorhandensein bedeutender Übertiefungen außerhalb der Alpenrandseen praktisch unmöglich. Nach der Theorie der randalpinen Rücksenkung wären auch die außerhalb der Alpenrandseen, wenn überhaupt vorhandenen, übertieften Bereiche, entweder mit lokaler tektonischer Absenkung oder mit interglazialer Flußerosion zu erklären.

Eine sachlich ernüchternde Richtigstellung in der Diskussion um die Wirksamkeit glazialer Tiefenerosion hat die Katastrophe beim Bau des Lötschbergtunnels (Nordseite, vom 24. Juli 1908) gebracht (vgl. dazu: Schweizerische Bauzeitung, LII/5:66, LII/6: 81, LII/ $8: 110,1908$ ). So ist auf tragische Art und Weise der Nachweis erbracht worden, daß auch in den inneralpinen Talabschnitten, orographisch oberhalb der Alpenrandseen, Felsbecken von gewaltiger Ubertiefung, wohl mit Lockermaterial („eiszeitlichem Schutt“) aufgefüllt, existieren. Im Falle der Gasterntalübertiefung haben spätere Sondierungen Lokkergesteinsmächtigkeiten von 200 bis 250 Meter nachgewiesen - die Füllung eines enormen Felskessels, wenn man sich die geomorphologische Situation bei der Klus (zwischen dem Gasterntal und dem Becken von Kandersteg) vergegenwärtigt! Außerhalb des Alpenkörpers und der Alpenrandseen hat eine erste Tiefensondierung auf grundwasserführende Lockergesteine im Becken von Belp den Beweis einer Mindestübertiefung von 88 Meter erbracht. Mit dem Nachweis einer Übertiefung um bedeutende Beträge auch im alpenferneren Mittelland ist die Diskussion um die Genese dieser mit Lockergesteinen aufgefüll- 
ten Wannen im Molasseland wieder aufgenommen und deren Entstehung im wesentlichen mit der wiederholten Vergletscherung als glaziale Tiefenerosion gesehen worden, ohne aber tektonische Bewegungen am Alpenrand, im Mittelland und bis an den Jurasüdfuß ganz von der Hand zu weisen (Beck 1921, Antenen 1936 und Schardt im „Geographischen Lexikon der Schweiz“, zitiert in ANTENEN 1936).

\subsection{Neue Forschungsergebnisse}

Erweiterte Gesichtspunkte zur Geologie der Alpenrandseen haben die Resultate der Seenprojekte der Geologischen Institute der Universität Bern (MatTer, Suesstrunk, Hinz \& Sturm 1971; Matter, Dessolin, Sturm \& Suesstrunk 1973; Sturm \& Matter 1972; Bodmer, Matter, Scheller \& Sturm 1973), der Universität Genf (Vernet, Horn, Badoux \& Scolari 1974) und der ETH Zürich gebracht (Finckh 1976; Finckh \& Kelts 1976). Dabei hat es sich gezeigt, daß die Übertiefung der Alpenrandseen im $N$ und der insubrischen Seen im S weit bedeutender ist als ursprünglich vermutet, daß insbesondere die insubrischen Seen gewaltige Becken darstellen. Aus den Angaben in Abb. 1 geht hervor, daß die zur Diskussion stehenden Seen in drei Gruppen eingeteilt werden können: 1. die insubrischen Seen mit Rinnentiefen bis über $500 \mathrm{~m}$ unter NN, 2. die „internen Alpenrandseen" (wie Genfer-, Brienzer-, Vierwaldstätter- und Walensee) mit Felsbeckentiefen unterhalb des Meeresspiegels bis ca. $300 \mathrm{~m}$ unter NN und 3. die „externen Alpenrandseen" (wie Thuner-, Zuger-, Zürich- und Bodensee) mit einer Felskote um NN.

Weitgehend ungeklärt ist in den Seebecken die Frage des Alters der tieferen und basalen Lockergesteine. Die vorliegenden Angaben beziehen sich vor allem auf spät- und postglaziale Akkumulationen. Aus den seismischen Untersuchungen geht hervor, daß die Lokkergesteinsabfolge eine komplizierte Auffüllung ist und daß generell vorbelastete, ältere, von nichtvorbelasteten, jüngeren, Sedimenten unterschieden werden können. Die Lockergesteinsmächtigkeiten in den Seebecken sind z. T. bedeutend: über $300 \mathrm{~m}$ im Thunersee (Matter, Suesstrunk, Hinz \& Sturm 1971: 516), bis über $2000 \mathrm{~m}(!)$ in den insubrischen Seen (FINCKH 1976: 89). - Diesen Lockergesteinsmächtigkeiten in den Seebecken sind jene in den „verlandeten“, zugeschütteten Becken, wie dem Becken von Belp mit $250 \mathrm{~m}$ und dem seeländischen Trog mit $270 \mathrm{~m}$ (ohne Berücksichtigung der Wassertiefe), gegenüberzustellen.

Interessant und von Bedeutung sind die Resultate des Präzisionsnivellements der Landestopographie (Landesnivellement), welche auf dem Höhenvergleich der von 1903 bis 1925 gemessenen Punkte mit der seit 1943 bis 1975 durchgeführten Nachmessungen beruhen. Auf die Fixpunktgruppe relativer Hebungs-, bzw. Senkungstendenz von Aarburg bezogen, ergibt sich eine Senkungstendenz von Oensingen westwärts dem Jurasüdfuß entlang ins Genferseebecken und Rhonetal aufwärts bis in die Gegend von St. Maurice mit den größten Senkungsbeträgen bis 0,6 mm/Jahr im Becken von Genf (Gubler 1976). Das heißt also, daß wir relativ auf Aarburg bezogen eine Kippbewegung des Mittellandes mit Abwärtstrend in der Westschweiz haben - eine Erscheinung, die während der Molassesedimentation und der obermiozänen, pliozänen und altpleistozänen Flußgeschichte des Mittellandes mehrmals mit verändertem Vorzeichen gespielt hat (Liniger 1966, 1967; Hofmann 1960; Mader 1977).

\section{Die Untersuchungsgebiete}

Geomorphologisch zeichnen sich im Gebiet des Kantons Bern zwischen Alpen und Jura drei Depressionen besonders ab: 1. das Aaretal Bern-Thunersee [gewissermaßen das nördliche Ende der Talfolge von Kander und Aare, in denen sich aus dem Gebiet der heutigen Gletscher (Kanderfirn, bzw. Ober- und Unteraar) ïbertiefte Becken aneinanderreihen 


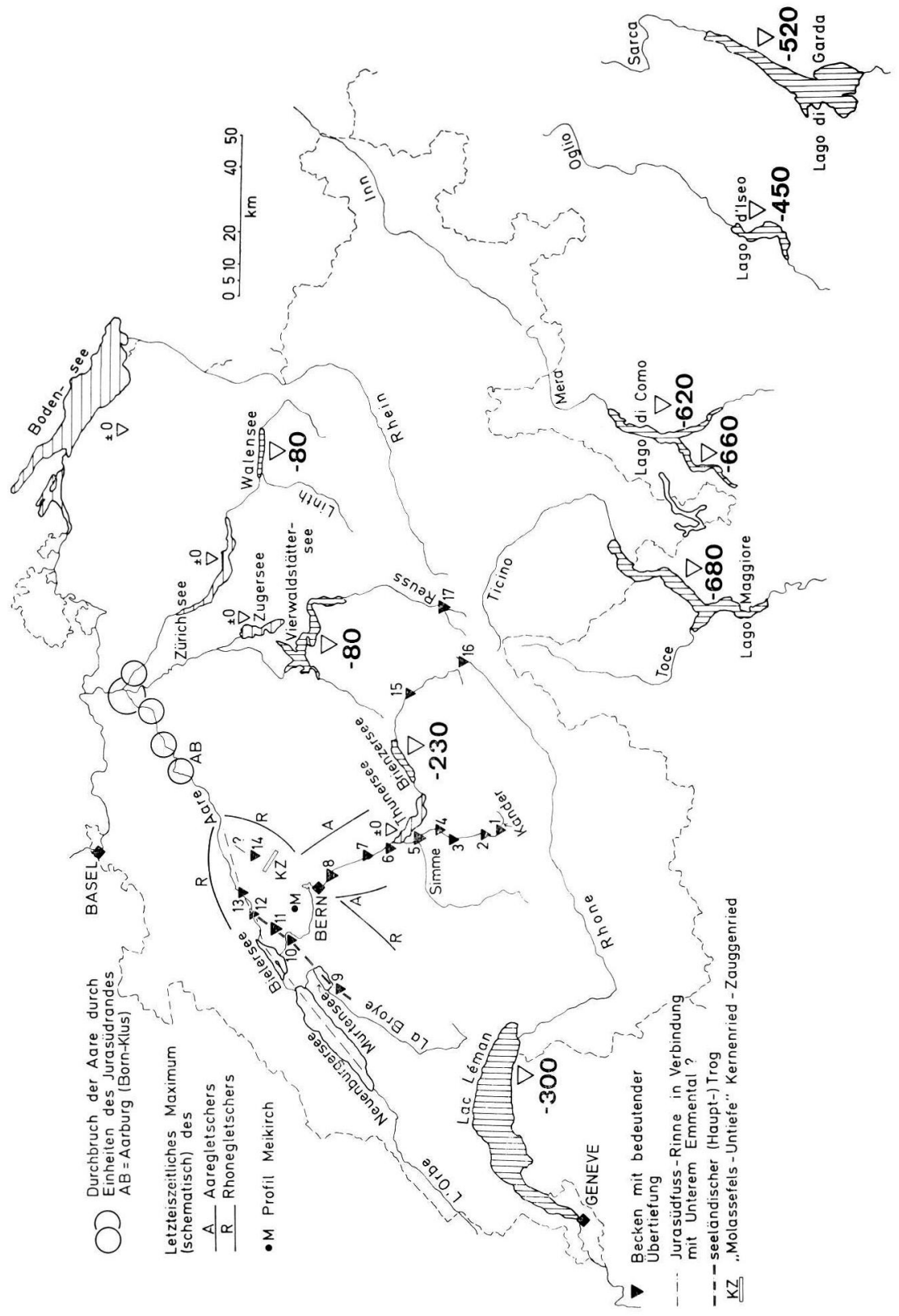

Abb. 1: Die Übertiefung der Schweizer Seen. - Mit besonderer Berücksichtigung der Felsbeckenfolge von Kander- und Aaretal und dem seeländischen Trog. Folgende Felsbecken sind besonders gekennzeichnet: 1. Gasterntal, 2. Becken von Kandersteg, 3. Becken Frutigen, 4. Becken von Reichenbach, 5. Becken von Wimmis, 6. Becken von Thun/Uttigen, 7. Becken von Kiesen, 8. B e c k e n v on B elp, 9. Ubertiefung bei Avenches, 10. Kallnach/Großes Moos, 11. Wor b e n/B u s s w i1, 12. Becken von Grenchen, 13. Ubertiefung bei Solothurn, 14. Becken im Unteren Emmental, 15. Becken von Innertkirchen, 16. Becken von Gletsch, 17. Becken von Andermatt. 
bis hinunter ins Becken von Belp], 2. das Bernische Seeland als Doppelrinne: MurtenseeAarberg-Lyss-Solothurn und Neuenburger-, Bielersee-Pieterlenmoos-Solothurn, 3. das Untere Emmental-Burgdorf-Gerlafingen-Wangen an der Aare (vgl. dazu Abb. 1).

Diese drei Depressionen bilden Bereiche mit bedeutend übertieften Molasse-Felsbecken, die mit einem Komplex eiszeitlicher Lockergesteine aufgefüllt sind.

Von Bedeutung in diesem Zusammenhang, besonders bei der Diskussion der glazialen Komponente in den übertieften Talabschnitten, ist die allgemeine Gletscherkonfiguration während der Vergletscherungen: die "Richtung" des Rhonegletschers von SW nach NE im seeländischen Trog und nahezu rechtwinklig dazu die Richtung des Aaregletschers (als vereinigte Oberländergletscher) aus dem Thunerseebecken durch das Aare-/Gürbetal nach N (zum Teil bis über Bern hinausreichend?). Das Felsbecken im Unteren Emmental läge ebenfalls im Einflußbereich des Rhonegletschers.

\subsection{Das Bernische Seeland}

Das Bernische Seeland ist als Niederung in seinem strukturellen Aufbau eine komplexe Abfolge von übertieften, langgezogenen Felsbecken und glazigen überformten Molassehöhen. In bezug auf die Molassestruktur stellen die aus miozänen Sedimenten aufgebauten Inselberge Synklinalstrukturen dar, währenddem die übertieften Talabschnitte in ihrer Längsausdehnung in die Molasseantiklinalen eingetieft sind. Der Längsverlauf der seeländischen Rinnen ist eindeutig durch die tektonische Struktur der Molasse gegeben: südwestlich der Linie Biel-Nidau-Jens-Lyss ist die Streichrichtung annähernd SW-NE, östlich dieser Linie erfolgt das Umbiegen auf WSW-ENE. Wichtige morphogenetische Bestimmungsgrößen sind in dieser Beziehung die Struktur des Jaissberges (zwischen dem heutigen Aarelauf bei Port und Jens-Worben gelegen) und des Büttenberges (östlich Biel, zwischen der Jurasüdfußrinne und der Haupt-Seelanddepression).

Im Auftrag des Wasser- und Energiewirtschaftsamtes (WEA) des Kantons Bern sind von 1972 bis 1976 im Bernischen Seeland durch Herrn Dr. P. Kellerhals und Herrn Dr. B. Tröhler, Geologen SIA, Bern, umfangreiche hydrogeologische Untersuchungen durchgeführt worden („Hydrogeologie Seeland“ 1976). Bereits oben wurde angeführt, daß die seeländische, übertiefte Rinne als Doppelrinne ausgebildet ist (Jurasüdfußrinne mit Bielerund Neuenburgersee und die Rinne des Großen Moos' mit Murtensee und altem Aarelauf Aarberg-Lyss-Büren a.A.). Aus den Untersuchungen des WEA geht klar hervor, daß die großen Übertiefungsbeträge in der südlichen der beiden Rinnen nachweisbar sind; diese wird im folgenden als seeländischer Trog (im Sinne von TroeHLER 1977) bezeichnet.

Im Großen gesehen ist der Felsverlauf als Basis des seeländischen Troges recht gut bekannt: bei Avenches südwestlich des Murtensees liegt die Molasse auf ca. $200 \mathrm{~m}$ ü. M., bei Kallnach auf ca. $320 \mathrm{~m}$ ü. M., dann folgt das tiefe Felsbecken südlich des Jaissberges mit der Felskote auf ca. $150 \mathrm{~m}$ ü. M. (vgl. Abb. 2). Nach den heute vorliegenden Daten folgt dann ostwärts das Ansteigen der Felsunterlage bis gegen $370 \mathrm{~m}$ ü. M. bei Solothurn (vgl. Angaben in Abb. 1).

Die Füllung des seeländischen Troges mit eiszeitlichen Lockergesteinen ist im einzelnen äußerst komplex, kann jedoch generell folgendermaßen als Abfolge schematisiert werden (vgl. auch Abb. 2):

- spät- und postglaziale Alluvionen (Flußablagerungen und Verlandungsbildungen)

- eine obere Grundmoräne

- hart gelagerte Seeablagerungen; monotone, siltig-tonig-sandige Sedimente mit unregelmäßig auftretenden Einlagerungen von Sand und Geröllagen, nach Kellerhals \& TroehleR (1976: 27) bis $300 \mathrm{~m}$ mächtig! 


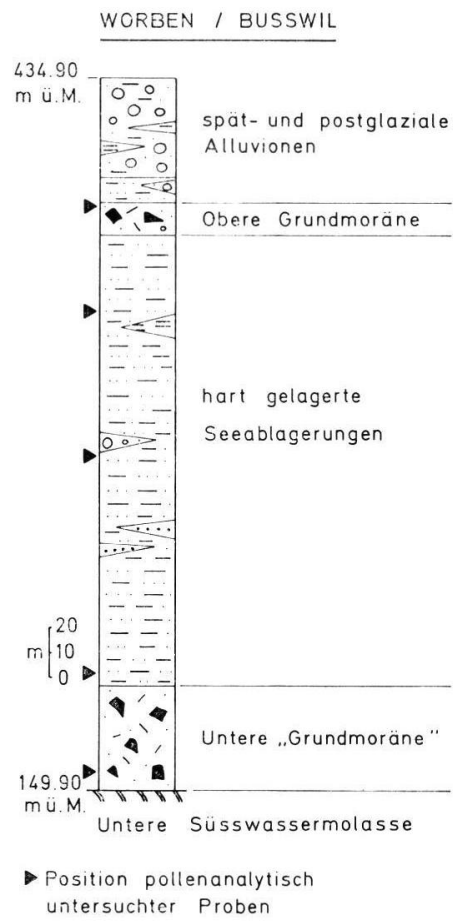

Abb. 2: Schematisches Lithologisches Profil der Bohrung Worben/Busswil nach Kellerhals \& Troehler (1976).

- eine untere Grundmoräne; der kaltzeitliche Charakter dieser Ablagerung ist durch pollenanalytische Untersuchungen von Herrn Prof. Welten (zitiert in Kellerhals \& TroeHLer 1976: 27) nachgewiesen, doch ist aus den Bohrprofilen die Ansprache als Grundmoräne oft weniger eindeutig.

Für ein direktes chronostratigraphisches Erfassen der Bildung der Erosionsbasis im Trog fehlen bisher die Grundlagen. Eine Alterszuweisung wird somit indirekt über die Datierung der basalen Lockergesteine angestrebt. Das kann im Seeland, abgesehen von einigen Streuproben aus der Bohrung Worben/Busswil (Abb. 2) nur indirekt über das Pollenprofil Meikirch (WELTEN 1976) geschehen (Situation in Abb.1). Ein chronostratigraphischer Vergleich zwischen den Ablagerungen im kleinen, ebenfalls übertieften Becken von Meikirch mit der seeländischen Trogfüllung ist zumindest vernünftig, wenn auch nicht auf einer Korrelation I. Ordnung im Sinne von Rutsch (1958: 116) beruhend, da einerseits anzunehmen ist, daß die wesentlichen Beiträge an die übertiefende Erosion im Molassehügelland und im Seeland gleichzeitig erfolgten und da andererseits ähnliche komplexe, und im Prinzip schematisch analog gliederbare Lockergesteinsabfolgen wie im seeländischen Trog auch bei Meikirch, also außerhalb der tiefsten Tröge des Seelandes, im "Plateaubereiche" vorhanden sind (vgl. Abb. 1, 2). - Da WeLten (1976) in Meikirch das Holstein-Interglazial pollenanalytisch nachgewiesen hat (über Mindel-spätglazialen Sedimenten) ist die grobe zeitliche Einstufung der Tiefenerosion im Seeland als mindestens Riß (im klassischen Sinne), wahrscheinlich jedoch älter, sinnvoll. Die mächtigen Seetone zwischen der Unteren und Oberen Grundmoräne im Profil Worben/Busswil (Abb. 2) werden 
im herkömmlichen Sinne als letztinterglazial (Riß/Würm) betrachtet. Diese Einstufung ist hier $\mathrm{nich} t$ gesichert, sondern es handelt sich nach den vorliegenden, allerdings spärlichen pollenanalytischen Untersuchungen um jeweils spätglaziale, frühinterstadiale Bildungen, die wahrscheinlich in rascher Folge in großen Mächtigkeiten abgelagert worden sind (Welten, schriftl. Mitt., Vorbereitung IGCP-Exkursion 1976).

Wenn man vorläufig vom Versuch einer genauen klimastratigraphischen (bzw. chronostratigraphischen) Einstufung der seeländischen Trogfüllung absieht, so steht fest, daß sowohl die tiefsten Felsbecken (wie auch kleinere Becken in Molassehügelland, wie Meikirch?) nach der tiefsten Erosion nicht einphasig aufgefüllt worden sind, sondern daß auf eine erste glaziale/spätglaziale Akkumulation erneute Tiefenerosion erfolgt ist, welche ein System von ineinandergeschachtelten, zum Hangenden hin zunehmend jüngere (und engere) Trogfolgen ergeben hat; dies trifft insbesondere zu, wenn man in die Morphogenese der tiefsten Rinnen auch die Lockergesteinsabfolgen der Rinnen-, bzw. Trogränder einbezieht.

\subsection{Das Untere Emmental}

Ebenfalls im Rahmen der siedlungswasserwirtschaftlichen Planung des Kantons Bern ist das Untere Emmental hydrogeologisch erkundet worden. Im Laufe dieser Untersuchungen ist auch hier ein bedeutendes, übertieftes Becken nachgewiesen worden, dessen Form und Ausdehnung jedoch noch nicht bekannt sind, insbesondere fehlt die Kenntnis seiner rein morphologischen Beziehungen zur Emmentalrinne oberhalb Burgdorf einerseits, zur Jurasüdfußrinne (als östliche Fortsetzung des seeländischen Troges) andererseits. - Die Bohrung RB 21 „Obergerlafingen“ des Wasser- und Energiewirtschaftsamtes (WEA) des Kantons Bern (Koord. 610.490/221.440) hat bei einer Geländehöhe von $469.70 \mathrm{~m}$ in 88 Meter Tiefe, also auf Kote $381.70 \mathrm{~m}$, den Felsuntergrund nicht erreicht. - Interessant, leider bis jetzt ohne litho- und palynostratigraphischen Bezug, ist das in der Bohrung RB 21 nachgewiesene Profil: die obersten $15.60 \mathrm{~m}$ werden von sandigem Kies mit Steinen, aber ohne wesentlichen Anteil an Feinanteilen (Ton und Silt) aufgebaut. Darunter folgen bis in die 88 Meter durchbohrte Tiefe Sande, vor allem Feinsande, mit reichlich bis viel Silt und wenig bis reichlich Ton. Die ganze Abfolge ist praktisch nicht weiter lithologisch gliederbar und von auffallender braun-, gelbgrauer Farbe - im Gegensatz zu den (blau-) grauen Seeablagerungen im seeländischen Trog und im Becken von Belp. Besonders unterhalb ca. $55 \mathrm{~m}$ zeichnen sich die hier erbohrten feinkörnigen Sedimente durch große Lagerungsdichte aus.

Das Felsbecken im unteren Emmental muß in zwei Richtungen weiter diskutiert werden:

1. In früheren Arbeiten (Nussbaum 1926, Gerber zit. in Beck \& Rutsch 1958: 29) wird als Fels- und Ausflußrinne aus dem Becken von Belp eine Verbindung nach NE via Urtenenlauf zum Unteren Emmental hin postuliert. Daß diese Felsrinnenverbindung offenbar nicht besteht, wird durch relativ oberflächennah anstehende Molasse (ca. $480 \mathrm{~m}$ ü. M., also nur 20-24 m unter Terrain) bei Kernenried-Zauggenried belegt (nach einer freundlichen mündlichen Mitteilung von Herrn Dr. R. Blau, WEA). Damit wird die postulierte Verbindung zwischen dem Becken von Belp und dem Unteren Emmental „abgeriegelt".

2. Im heutigen orographischen Sinne flußabwärts hat das Felsbecken des unteren Emmentals, östlich Solothurn in Verbindung mit der Jurasüdfußrinne, entweder als einfache Rinne oder aber als Trogfolge, eine weitere Ausdehnung dem Jurasüdfuß entlang. Bei Aarburg, also dem relativen Ruhe- und Ausgangspunkt des Landesnivellements, verläßt die Aare das Mittelland und tritt durch eine Klus durch die Born-Antiklinale in das Jura- 
gebirge ein. Die Felskote der Aare an diesem Punkt, wo die Flußgeschichte von Mittelland und Jura direkt miteinander in Beziehung treten, liegt auf $361.70 \mathrm{~m}$ ü. M., in bezug auf die Felskote bei Solothurn nur ca. $10 \mathrm{~m}$ tiefer, in bezug auf die Felskote bei Gerlafingen, wo diese sicher unter $381.70 \mathrm{~m}$ ü. M. liegt, mindestens nur ca. $20 \mathrm{~m}$ tiefer, wobei nicht auszuschließen ist, daß der Fels im Becken des Unteren Emmentales noch bedeutend unter dem Schwellenwert von $361.70 \mathrm{~m}$ von Aarburg liegt. Das Gefälle der heutigen Aare verläuft also in deutlichem Gegensatz zum Verlauf der Felsoberfläche.

\subsection{Das Aaretal Bern-Thunersee, insbesondere das Becken von Belp}

Die Haupttalfolge des Berner Oberlandes, die Aarerinne vom Oberhasli, durch Brienzer- und Thunersee mit den hauptsächlichsten Tributärtälern von Kander und Simme, findet im Becken von Belp, also ca. $25 \mathrm{~km}$ außerhalb des Alpenrandes, ihren vorläufigen, morphologischen Abschluß. Es ist in diesem Zusammenhang sinnvoller, weder von einer Aare- noch von einer Kanderrinne, sondern von einer Felsbeckenfolge in diesen zwei bedeutendsten Tälern zu sprechen. Die wichtigsten dieser übertieften Talabschnitte, welche die Felsbeckenfolge aufbauen, sind in Abb. 1 schematisch dargestellt. Nördlich des Beckens von Belp ändert dieser Felsbeckenfolge-Charakter des Aaretals grundsätzlich (oder ist noch nicht erkannt worden?).

Die primäre Anlage der Berner Oberländer Täler, wie Aare-, Kander- und Simmental, entlang tektonisch bedeutender Bewegungs- und Grenzzonen, geht aus dem Aufbau des Alpenkörpers hervor. Besonders hinzuweisen ist in diesem Zusammenhang auf die Inkongruenz in Bau und paläogeographischer Provenienz der Alpenrandelemente östlich und westlich des Thunersees. Diese Inkongruenz der tektonischen Elemente östlich und westlich des Aaretals läßt sich z. T. bis hinaus in die subalpine Molasse verfolgen. Wie weit hinaus im Molassevorland aber dieser tektonische Grundton in der Aaretalanlage sich durchpaust, muß Spekulation bleiben (vgl. dazu Matter, Suesstrunk, Hinz \& Sturm 1971 und Schluechter 1973, 1976).

Aus den bisherigen Untersuchungen geht hervor, daß im Brienzersee die tiefste Felskote der Aaretalrinne (zwischen dem Oberhasli und Bern) nachweisbar ist $=-230 \mathrm{~m}$ unter NN (Matter, Dessolin, Sturm \& Suesstrunk 1973). „Flußabwärts“ schließt das Thunerseebecken mit einer Felskote auf ca. $0 \mathrm{~m}$ NN an (MatTer, Suesstrunk, Hinz \& STURM 1971). Nach Beck (1938) folgt dann im Bereich unterer Thunersee/Schwemmfächer von Kander und Zulg der Anstieg des Felsuntergrundes um 150 bis $250 \mathrm{~m}$ um dann, ebenfalls nach BECK (1938), im mittleren Aaretal und im Becken von Belp konstante Gefällsverhältnisse aufzuweisen. Durch die Bohrung im Marzili des WEA wissen wir heute, daß die Molasse auf Kote ca. $230 \mathrm{~m}$ ü. M. ansteht, durch die Bohrung Hunziken (verbunden mit geophysikalischen Untersuchungen), daß der Fels auf ca. $250 \mathrm{~m}$ ii. M. zu erwarten ist, das heißt, daß die Felssohle des Beckens von Belp (zwischen diesen beiden Bohrungen) annähernd gleiche Gefällsverhältnisse aufzuweisen scheint, wie die heutige Terrainoberfläche (vgl. dazu Abb. 3).

Die folgenden Ausführungen konzentrieren sich auf das Becken von Belp, einmal weil dieser Talabschnitt als Kernstück in der Diskussion der Übertiefung des Aaretals aufzufassen ist und zweitens weil keine Tiefendaten aus dem Abschnitt Hunziken-Thunersee vorliegen.

Unsere heutige Kenntnis über das Felsbecken Hunziken-Belp-Marzili südlich von Bern und dessen Lockergesteinsfüllung beruht im Wesentlichen auf den beiden Tiefbohrungen $\mathrm{B} 1$ und $\mathrm{H} 1 \mathrm{a}$ (s. Abb. 3 und 4), sowie dazwischenliegenden Zusatzbohrungen ge- 
ringerer Tiefe. Bei den beiden Tiefbohrungen handelt es sich um Spühlbohrungen, so daß die eingetragenen lithologischen Gliederungen mit etwas Vorsicht weiterverwendet werden sollten (nach Bohrprofilen des WEA, die mir freundlicherweise von Herrn Dr. R. Gees zur Verfügung gestellt worden sind).

\section{NW}

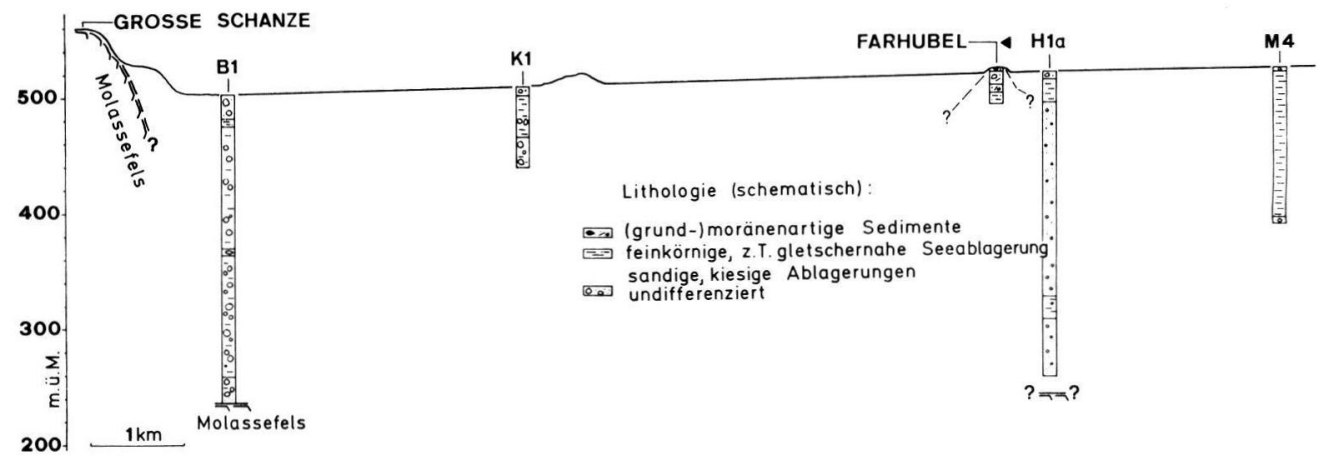

Abb. 3: Längsprofil durch das Becken von Belp von der Stadt Bern (Große Schanze, nördlich des Hauptbahnhofes) bis südlich von Hunziken (Hla = Bohrung 1a "Hunziken“). Die Lithologie der Beckenfüllung ist vereinfacht und schematisch dargestellt.

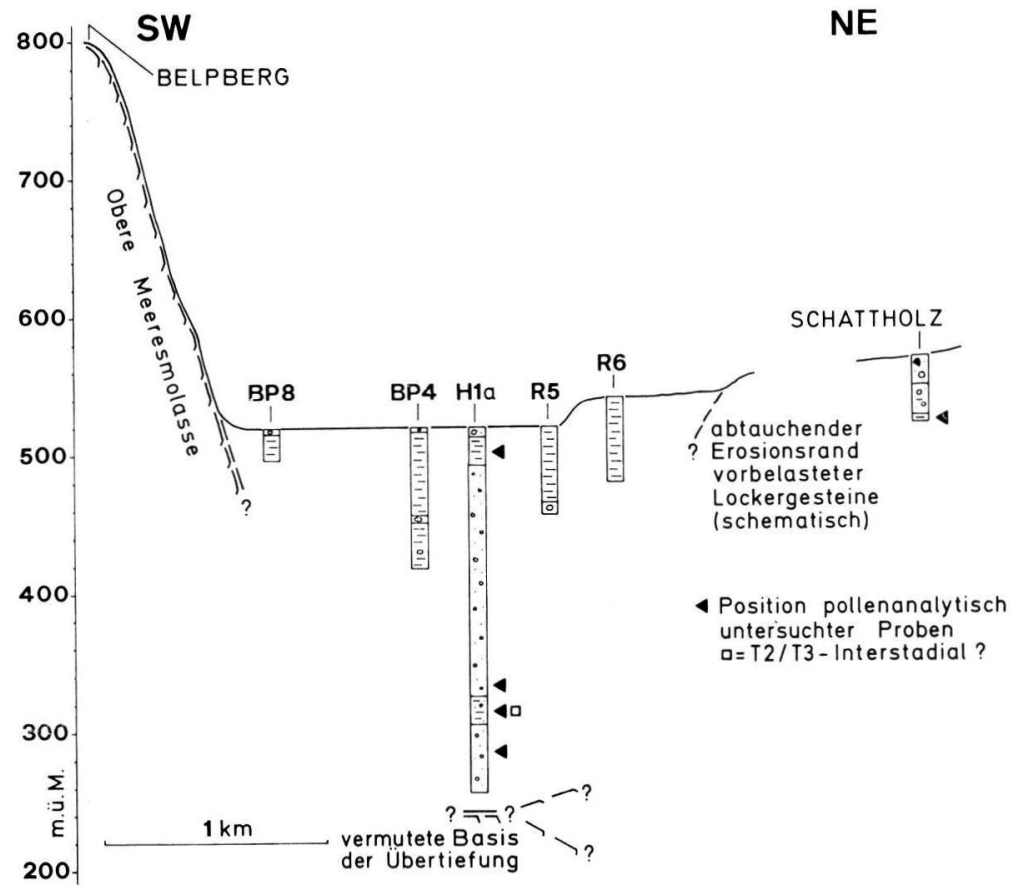

Abb. 4: Querprofil durch das südliche Becken von Belp (Belpberg-Schattholz, östlich von Rubigen). Die lithologische Darstellung der Bohrprofile ist vereinfacht und schematisch. 
Auffallend ist, wie in beiden Tiefbohrungen über bedeutende Mächtigkeiten Grobsand (H1a) oder Feinkies-, bzw. Kiesablagerungen mit reichlich Feinanteilen nachgewiesen worden sind, währenddem gerade im Querschnitt von Hunziken und südlich davon (M4, Abb. 3) mächtige feinkörnige Sedimente erbohrt worden sind. Zu bemerken ist aber, daß in keiner der eingetragenen Bohrungen eine tiefliegende Grundmoräne (wie im Seeland, Abb. 2) nachgewiesen worden ist.

Für die stratigraphische Gliederung dieser Beckenfüllung können folgende drei Bohrungen herangezogen werden: $\mathrm{H} 1 \mathrm{a}$, Farhubel und Schattholz; erstens weil dort vorläufige palynostratigraphische Untersuchungsresultate von Herrn Prof. Welten vorliegen, zweitens weil Farhubel und Schattholz auch lithostratigraphisch einigermaßen mit Oberflächenaufschlüssen des Aaretalrandes verglichen werden können:

Im Farhubelprofil finden wir als oberste, eindeckende Schicht eine geringmächtige, tonige graublaue Grundmoräne, die von verbraunten Schottern mit reichlich Silt und Ton unterlagert wird. WELTEN konnte nun zeigen, daß die eindeckende Grundmoräne wohl aufgearbeitetes T2/T3 - interstadiales (oder Eem - ?) Material enthält, also aus einem altersgleichen Horizont aufgearbeitet worden ist, wie er in Bohrung H1a mit der zweituntersten Probeentnahmestelle bezeichnet ist. Die Frage, ob das T2/T3-Interstadial im Sinne des Thalgut-Interstadials in Wirklichkeit eine Interstadial- oder Interglazialzeit repräsentiert (vgl. WELTEN 1976) sei in diesem Zusammenhang nicht diskutiert; ebenfalls wird hier nicht auf die Frage der Position des Eem-Interglazials im klassischen Schema Günz-Mindel-Riß-Würm und auf die Frage, ob Riß = "größte Vergletscherung der Schweizeralpen" = T2 (sensu WeLTEN) eingegangen.

Wichtig für den Versuch einer chronostratigraphischen Einstufung der Belper Beckenfüllung sind die palynostratigraphischen Resultate von der Basis der Bohrung Schattholz (Abb. 4): Welten hat dort in Seetonen eine Pollengemeinschaft von interglazialem Charakter nachgewiesen, die er mit Fragezeichen in ein „älteres Interglazial“ (kaum ins Eem) stellen möchte. - Wenn wir bedenken, daß nordöstlich der Bohrung Schattholz (Abb. 4) erst in einem Kilometer Entfernung die Molasse wieder die Geländeoberfläche bildet und $\mathrm{da}$ in den Bohrungen R6, R5, H1a, BP4 und BP8 durchschnittlich die obersten 30-40 m locker gelagert (die in H1a nach WELTEN = spätglazial) sind, so erinnern wir uns wieder an die morpho-/lithostratigraphische Situation im Seeland: nach der ursprünglichen Hauptphase der Tiefenerosion erfolgte auch im Aaretal eine Akkumulation, die noch heute randlich des Haupttroges und in großer Tiefe (?) in demselben reliktisch nachweisbar ist; zeitlich folgt darauf eine ineinandergeschachtelte Trogfolge, deren Erosionsbasis die „alte Basis", nämlich die tiefste Felsrinne, nicht mehr (?) erreichte. So kann, ohne die Ursache der ursprünglichen Tiefenerosion zu erklären, festgehalten werden, daß mindestens seit der vorletzten Eiszeit (Riß im klassischen Sinne), wahrscheinlich aber seit einem Ereignis vor der „Riß“-Eiszeit die Übertiefung im Fels zum Stillstand gekommen ist und daß die folgenden Vergletscherungen im wesentlichen durch Akkumulationen belegt sind. Schematisch und z. T. vereinfacht gesprochen, finden wir sowohl im Aaretal (Abb. 4) als auch im seeländischen Trog vom Trogrand nach innen, wie vom Liegenden zum Hangenden, in der Trogfüllung zunehmend jüngere Ablagerungen.

\section{Zusammenfassende Bemerkungen und Ausblick}

1. Die primär tektonische Anlage der Berner Oberland Täler (Aare-, Kander- und Simmental) ist im Inneren des Alpenkörpers bis hinaus in die subalpine Molasse nachgewiesen. Im inneralpinen Bereich der Tal- bzw. Felsbeckenfolge ist anzunehmen, daß neben der glazialen Tiefenerosion subrezente und rezente Krustenbewegungen an der Genese übertiefter Talabschnitte wesentlich beteiligt sind, wie das ECKARDT (1957) für das Becken von Andermatt gezeigt hat. 
2. Im Berner Querschnitt des schweizerischen Mittellandes lassen sich zwei nahezu senkrecht zueinander verlaufende Felsbeckenfolgen voneinander unterscheiden:

- die Beckenfolgen der Oberlandtäler mit der Konfluenz nördlich Thun und dem nördlichen Abschluß im Becken von Belp = im Bereiche der eiszeitlichen, vereinigten Oberländergletscher gelegen

- der seeländische Trog (mit der bei Grenchen tributären Jurasüdfußrinne) und dem Becken im Unteren Emmental. Dazu gehören auch kleinere übertiefte Becken im Molassehügelland nördlich von Bern $=$ im Bereiche des eiszeitlichen Rhonegletschers gelegen.

Eine Basisverbindung (als Erosionsrinne) zwischen den beiden übertieften Bereichen ist bis jetzt nicht nachgewiesen worden. Im Gegensatz dazu steht der heutige Lauf der Aare.

3. In der Bohrung Marzili (B1, Abb. 3) ist in großer Tiefe artesisches Wasser erbohrt worden, dessen Alter am Physikalischen Institut der Universität Bern, unter der Leitung von Herrn Prof. Oeschger (1977) mit $5000{ }^{14} \mathrm{C}$-Jahren bestimmt worden ist. Der Nachweis von fossilem, gefangenem Wasser im Becken von Belp kann nicht als Beweis für das Fehlen eines basalen Ausflusses, zumindest aber als Hinweis gelten.

Damit in Ubereinstimmung steht eine Angabe von Kellerhals (1977), wonach kleinere Erosionsrinnen in der Molasse in der Umgebung von Bern ein Gefälle zum Becken von Belp hin aufweisen.

4. Die Datierung der maximalen Ausräumung hängt im wesentlichen von der chronostratigraphischen Einstufung der Lockergesteine in den betrachteten Talabschnitten ab. Wenn wir mit WeLteN annehmen, daß sowohl in der Bohrung Schattholz als auch in der Kanderschlucht (dort offenbar im Hangenden der Grundmoräne im Hani, Schluechter 1973) „ältere interglaziale Bildungen“, also prae-Eem, möglicherweise vorhanden sind, dann wird die Ausräumung der tiefsten Rinne im Aaretal Bern-Thun als sicher praerißeiszeitlich (im klassischen Sinne) aufzufassen sein. Was sicher feststeht, ist, daß sowohl im Aaretal als auch im Seeland die eiszeitlichen, morphogenetischen Ereignisse seit mindestens der vorletzten Eiszeit im wesentlichen akkumulativ und nicht mehr nur erosiv gewirkt haben; zudem sind die Aufschüttungen in den übertieften Becken sicher mehrphasig erfolgt.

5. Die bis heute vorliegenden palyno- und klimastratigraphischen Daten weisen darauf hin, daß die oft sehr mächtigen Seeablagerungen (Seetone sensu lato) in den übertieften Becken aus den spätglazialen Zeiten stammen und in rascher Folge geschüttet worden sind. Jedenfalls ist das oft postulierte interglaziale Alter dieser Bildungen für die weitaus mächtigsten Abschnitte nicht bewiesen worden.

6. Ein Deutungsversuch der Ursache der Ubertiefung im Seeland sollte zumindest als Möglichkeit das Mitwirken von Senkungsvorgängen, wie sie mit dem Landesnivellement (Gubler 1976) nachgewiesen worden sind, gelten lassen. - Im Laufe der Monate November-Dezember 1977 sind im Grauholz (östlich von Bern) Sondierbohrungen für ein Vorprojekt der Schweiz. Bundesbahnen abgeteuft worden. Bei einer Bohrung sind bedeutende Bodengasaustritte $\left(\mathrm{CO}_{2}, \mathrm{CH}_{4}\right.$ ?) registriert worden. Nach den Forschungen der Tübinger Geologen Prof. Ernst und Mitarbeiter sind solche Gasaustritte an junge Störungszonen in der Molasse gebunden (MAder, briefl. Mitt. vom 2.3.1971). Diese Bohrung liegt genau auf der Linie Gurten — nördliche Begrenzung des Beckens von Belp-Grauholz, welche bisher immer als Erosionskante des Rhonegletschers erklärt worden ist.

Die Versuche einer genetischen Erklärung des Beckens von Belp erhalten somit konkrete, neue Aspekte. - Die Deutung der Entstehung des Beckens im Unteren Emmental muß noch offen bleiben, bis dessen Morphologie besser bekannt ist. Seine Ansprache als 
glaziales Konfluenzbecken (?, Aare- und Rhonegletscher/Emmengletscher während der größten Vergletscherung) ist vorläufig eine vernünftige Annahme.

7. Aus den Resultaten des Landesnivellements geht hervor (GUbler 1976), daß das westliche Molassebecken (westlich von Aarburg) eine Senkungstendenz aufweist. In diesem Zusammenhang sei, ohne jegliche Deutung, folgende Gegenüberstellung erwähnt: in der Ostschweiz liegen die ältesten sicher pleistozänen Lockergesteine in Form der Deckenschotterkomplexe in gehobener morphostratigraphischer Position vor. Im Aaretalquerschnitt liegen die bisher ältesten nachgewiesenen eiszeitlichen Lockergesteine an der Basis der pleistozänen Abfolge (Schotter von Bümberg, Grundmoräne im Hani, Schluechter 1973), unter den vor- und letzteiszeitlichen Akkumulationen. Sowohl für die Schotter von Bümberg als auch für die Grundmoräne im Hani ist frühes vorletzteiszeitliches Alter (Riß im klassischen Sinne) als Mindestalter sicher, wesentlich höheres Alter wahrscheinlich (SCHLUeCHTER 1973).

8. Eine Nachsymposiumsdiskussion in Rosenheim mit Herrn Dr. van Husen, Wien, hat den Gedanken aufkommen lassen, daß die Felsbecken in unseren Alpentälern und im Vorland ihre Anlage einer Gletscherzungenposition verdanken; dabei wäre der Gletscher über längere Zeiten in dieser Gleichgewichstlage geblieben und so wäre es zu einer Zungenbeckenbildung durch die gewöhnliche interne Fließ- und Erosionstätigkeit des Eises gekommen. Es wäre dies, bei bestimmter Gletscherausdehnung, eine in das Alpenvorland verlagerte Cirque-Bildung.

9. Wenn wir heute unser Wissen zusammenstellen über die Ursache der Bildung übertiefter Talabschnitte, dann sehen wir, daß wir viele wertvolle Daten zusammengetragen haben, um die Diskussionen der Jahrhundertwende in einem sachlicheren Licht zu sehen. Um aber das Wesen der Übertiefung voll zu erfassen, um den letzten Schritt aus diesem Fragenkreis hinaus gehen zu können, müssen wir weiterhin der alten Goldgräberlosung folgen: „Dig a little more!“

\section{Verdankung}

Herrn Prof. H. J. Lang, Vorsteher IGB-ETHZ, danke ich verbindlichst für sein stetes Interesse an meiner Arbeit im Quartär. - Herrn Prof. Dr. M. Welten, Bern, danke ich herzlich für seine Bereitschaft, unsere gemeinsamen wissenschaftlichen Probleme zu diskutieren. Herrn Prof. Dr. B. Frenzel, Stuttgart, und Herrn Prof. Dr. G. Furrer, Zürich, verdanke ich manche Anregung zu weiterer Forschungstätigkeit.

Es ist mir ein aufrichtiges Anliegen, folgenden Herren für das Überlassen von Grundlagenmaterial für die siedlungswasserwirtschaftliche Planung des Kantons Bern und für gemeinsame Diskussionen im Büro und im Feld zu danken: Herrn Dr. V. Blau, WEA, Bern; Herrn Dr. R. Gees, WEA, Bern; Herrn Dr. P. Kellerhals, Bern und Herrn Dr. B. Tröhler, Bern.

Herrn Dr. M. Sturm, EAWAG-ETHZ, danke ich für seine mannigfachen guten (Literatur-)Hinweise und Herrn Dr. D. van Husen, Wien, für seine anregenden Diskussionsbeiträge.

\section{Schriftenverzeichnis}

Antenen, F. (1936): Geologie des Seelandes. - Biel (Heimatkundekommission).

BECK, P. (1921): Grundzüge der Talbildung im Berner Oberland. - Eclogae geol. Helv., 16/2: 139-176; Basel.

- (1938): Bericht über die außerordentliche Frühjahrsversammlung der Schweiz. Geol. Ges. in Thun. - Eclogae geol. Helv., 31/1: 173-198; Basel.

- \& Rutsch, R. F. (1958): Geologischer Atlas der Schweiz, Erläuterungen zu Atlasblatt 21. Bern (Kümmerly \& Frey). 
Bodmer, R., Matter, A., Scheller, E. \& Sturm, M. (1973): Geologische, seismische und pollenanalytische Untersuchungen im Bödeli bei Interlaken. - Mitt. naturforsch. Ges. Bern, N.F., 30: $51-62$; Bern.

ECKardt, P. M. (1957): Zur Talgeschichte des Tavetsch, seine Bruchsysteme und jungquartären Verwerfungen. - Mitt. Geol. Inst. ETH u. Univ. Zürich, C/73, 96 S., Zürich.

FINCKH, P. (1976): Wärmeflußmessungen in Randalpenseen. - Mitt. Geol. Inst. ETH u. Univ. Zürich, N.F., 216 (ETH-Diss. Nr. 5787), 104 S.; Zürich.

- \& Kelts, K. (1976): Geophysical investigation into the nature of preholocene sediments of Lake Zurich. - Eclogae geol. Helv., 69/1: 139-148; Basel.

Frueh, J. (1906): Úber Form und Größe der Glazialerosion (L'érosion glaciaire et la topographie actuelle). - Eclogae geol. Helv., 9: 732-733; Basel.

Gogarten, E. (1910): Uber alpine Randseen und Erosionsterrassen, im besonderen des Linthtales. - Peterm. geogr. Mitt., Erg.-heft No. 165, 80 S.; Gotha.

Gubler, E. (1976): Beitrag des Landesnivellements zur Bestimmung vertikaler Krustenbewegungen in der Gotthard-Region. - Schweiz. mineral. petrogr. Mitt., 56: 675-678; Zürich.

Heım, A. (1885): Handbuch der Gletscherkunde. Bibliothek Geographischer Handbücher. - Stuttgart (Engelhorn).

- (1919): Geologie der Schweiz; Band 1, Molasseland und Juragebirge. - Leipzig (Tauchnitz).

Hofmann, F. (1960): Materialherkunft, Transport und Sedimentation im schweizerischen Molassebecken. - Ber. ü. d. Tätigkeit d. St. Gall. naturwiss. Ges., Bd. 76, 1956/58: 49-76; St. Gallen.

Kellerhals, P. (1977): Einige Hinweise auf das Molasserelief nördlich und westlich von Bern. Schweiz. natf. Ges., Vortrag an der 157. Jahresversammlung in Bern, 6.-9. Oktober 1977.

- \& Troenler, B .(1976): Grundlagen für die siedlungswasserwirtschaftliche Planung des Kantons Bern. Hydrogeologie Seeland, 149 S. - Bern (Wasser- und Energiewirtschaftsamt des Kantons Bern, WEA).

Liniger, H. (1966): Das plio-altpleistozäne Flußnetz der Nordschweiz. - Regio Basiliensis, VII/2: 158-177; Basel (Helbing \& Lichtenhahn).

- (1967): Pliozän und Tektonik des Juragebirges. - Eclogae geol. Helv., 60/2: 407-490; Basel.

Matter, A., Suesstrunk, A. E., Hinz, K. \& Sturm, M. (1971): Ergebnisse reflexionsseismischer Untersuchungen im Thunersee. - Eclogae geol. Helv., 64/3: 505-520; Basel.

- , Dessolin, D., Sturm, M. \& Suesstrunk, A. E. (1973): Reflexionsseismische Untersuchungen im Brienzersee. - Eclogae geol. Helv., 66/1: 71-82; Basel.

Nussbaum, F. (1927): Das Moosseetal, ein diluviales Fluß- und Gletschertal. - Mitt. naturforsch. Ges. Bern a. d. Jahre 1926: 122-169; Bern.

Oeschger, H. (1977): Isotopische Methoden der Quartärforschung. - Schweiz. natf. Ges., Vortrag an der 157. Jahresversammlung in Bern, 6.-9. Oktober 1977.

Rutsch, R. F. (1958): Das Typusprofil des Helvétien. - Eclogae geol. Helv., 51/1: 107-118; Basel.

Schardt, H.: Geographisches Lexikon der Schweiz. - Zitiert in Antenen, F. (1936).

Schluechter, Ch. (1973): Geologische Untersuchungen im Quartär des Aaretals südlich von Bern Stratigraphie, Paläontologie, Sedimentologie). - Diss. Univ. Bern, 307 S.; Bern. [Unveröff.].

- (1976): Die lithostratigraphische Gliederung der letzteiszeitlichen Ablagerungen zwischen Bern und dem Thunersee. In: Frenzel, B. (Hrsg.): Führer zur Exkursionstagung des IGCPProjektes 73/1/24 "Quaternary Glaciations in the Northern Hemisphere" vom 5.-13. 9. 1976 in den Südyogesen, im nördlichen Alpenvorland und in Tirol. - Stuttgart, Hohenheim.

Schweizerische Bauzeitung (1908): Monatsausweise und Berichte über die Arbeiten am Lötschbergtunnel. — LII/5: 66, LII/6: 81, LII/8: 110; Zürich.

Staub, R. (1938): Prinzipielles zur Entstehung der alpinen Randseen. - Eclogae geol. Helv., 31/2: 239-258; Basel.

Sturm, M. \& MatTer, A. (1972): Geologisch-sedimentologische Untersuchungen im Thuner- und Brienzersee. - Jb. Thuner- u. Brienzersee, 1972: 3-23; Interlaken.

Troenler, B. (1977): Der seeländische Trog und seine quartäre Füllung. - Schweiz. natf. Ges., Vortrag an der 157. Jahresversammlung in Bern, 6.-9. Oktober 1977.

Vernet, J.-P., Horn, R., Badoux, H. \& Scolari, G. (1974): Etude structurale du Léman par sismique réflexion continue. - Eclogae geol. Helv., 67/3: 515-529; Basel.

Welten, M. (1976): Das jüngere Quartär im nördlichen Alpenvorland der Schweiz auf Grund pollenanalytischer Untersuchungen. In: FrenzeL, B. (Hrsg.): Führer zur Exkursionstagung des IGCP-Projektes 73/1/24 "Quaternary Glaciations in the Northern Hemisphere" vom 5.-13. 9.1976 in den Südvogesen, im nördlichen Alpenvorland und in Tirol. - Stuttgart, Hohenheim.

8 Eiszeitalter u. Gegenwart 
